\title{
Monitoring of Infant Feeding Behavior Using a Jaw Motion Sensor
}

\author{
Muhammad Farooq ${ }^{1}$, Paula C Chandler-Laney ${ }^{2}$, Maria Hernandez-Reif ${ }^{3}$ \\ and Edward Sazonov ${ }^{*}$ \\ ${ }^{1}$ Department of Electrical and Computer Engineering, \\ ${ }^{3}$ Department of Human Development \& Family Studies, University of Alabama, \\ Tuscaloosa, AL USA \\ ${ }^{2}$ Department of Nutrition Sciences, University of Alabama at Birmingham, \\ Birmingham AL USA
}

Submitted January 2014. Accepted for publication November 2014.

\begin{abstract}
Rapid weight gain during infancy increases the risk of obesity. Given that infant feeding may contribute to rapid weight gain, it would be useful to develop objective tools which can monitor infant feeding behavior. This paper presents an objective method for examining infant sucking count during meals. A piezoelectric jaw motion sensor and a video camera were used to monitor jaw motions of 10 infants during a meal. Videotapes and sensor signals were annotated by two independent human raters, counting the number of sucks in each 10 second epoch. Annotated data were used as a gold standard for the development of the computer algorithms. The sensor signal was de-noised and normalized prior to computing the per-epoch sucking counts. A leave-one-out cross-validation scheme resulted in a mean error rate of $-9.7 \%$ and an average intra-class correlation coefficient value of 0.86 between the human raters and the algorithm.
\end{abstract}

Keywords: wearable sensors, obesity, sucking count, sucking frequency, feeding behavior, jaw motion, breast-fed, bottle-fed, infants

\section{INTRODUCTION}

Obesity is caused by excessive accumulation of body fat. Previous research suggests that infancy may be a critical period during which lifetime risk for obesity develops. Infants fed formula from bottles experience more rapid weight gain (for a systematic review see [1]) and have greater life-time risk for obesity [2,3] in comparison to breastfed infants. In addition, bottle-fed infants have greater energy intake, and differing meal patterns and within-meal behavior as compared to breast-fed infants $[4,5]$. Independent of the mode of feeding, rapid weight gain in the first six months of life is also associated with subsequent lifetime risk for obesity [6-14]. Vigorous sucking at 3 months of age

*Corresponding author: Edward Sazonov, PhD, Associate Professor, Department of Electrical and Computer Engineering, University of Alabama, Tuscaloosa, AL-35401, USA. Tel: +1-205-348-1981, Fax: +1-205-3486959, Email: esazonov@eng.ua.edu. 
is positively associated with adiposity at 12 months of age [15]. Finally, excess energy intake during infancy is a primary determinant of rapid weight gain and subsequent body size [16]. Together, these results suggest that feeding behavior during infancy may play an important role in the development of obesity. However, methods to monitor feeding behavior and to assess energy intake among infants are limited.

This paper presents a novel approach for monitoring infant sucking count (number of sucks) during meals. If the sucking count can be accurately determined, it will be possible to derive sucking vigor or rate of sucking (sucking frequency) which, as mentioned above, is associated with adiposity in early life. With further development, this tool might also facilitate efficient characterization of other feeding behaviors such as meal duration, frequency of intake, and volume consumed. Use of a jaw motion sensor was previously found to provide accurate and objective monitoring of ingestive behavior in adults [17-21]. Since infants use their jaws during sucking (both bottle-fed and breast-fed), the jaw motion sensor may be a useful tool to capture sucking count during feeding episodes, especially in breast-fed infants. To the best of the authors' knowledge, no previous study has reported the use of a jaw sensor to monitor feeding behavior in infants.

The goal of this study was to evaluate the technical feasibility of using a non-invasive jaw motion sensor to monitor sucking count and thereby sucking frequency (number of sucks per unit time (seconds) of infants during a meal. Data from the jaw sensor were compared to data obtained by human observation to examine the accuracy of the sensorderived estimates of the sucking counts for both breast-fed and bottle-fed infants.

\section{METHODS}

\subsection{Participants}

Ten infants were recruited for this study. Infants were eligible for inclusion if they were healthy and between 2 and 5 months of age, with a current weight-for-length not less than the $5^{\text {th }}$ percentile based on the Centers for Disease Control and Prevention (CDC) growth curves [22]. Infants were excluded if they were less than 37 weeks of gestation and/or less than $2500 \mathrm{~g}$ at birth. The Institutional Review Boards (IRBs) at the University of Alabama at Birmingham (UAB, site for data collection) and the University of Alabama (UA, site for data analysis) approved this study. Mothers of infants provided informed consent before data collection began. The relatively small sample size of this study was stipulated by the pilot nature of the conducted work.

\subsection{Protocol}

Participants and their mothers came to the UAB Child Health Research Center (CHRU), located within the Children's of Alabama hospital facility, to complete the study protocol. Each infant came to the laboratory for one visit which was scheduled to occur at a time when the infant was expected to be hungry, and not less than 2 hours following the previous meal. After informed consent was obtained, infant weight (without clothing) and length (supine) was measured using standard clinical procedure. Infants then underwent a weighed, timed, and videotaped meal test while wearing the jaw motion sensor. After the completion of the meal, infant body composition was assessed by air displacement plethysmography (PeaPod®; Cosmed Inc., Concord, CA). 


\subsection{The Meal}

All infants were weighed to the nearest 0.1 gram, while wearing a clean diaper only, on the weighing scale of the PeaPod ${ }^{\circledR}$. For bottle-fed infants, the prepared bottle was also weighed to the nearest 0.1 gram prior to the meal. The mother sat in an armchair to breast- or bottle-feed her infant. The mother held her infant in a side-lying position, with the infant's head supported in the crook of her bent arm. The jaw sensor (described below) was adhered directly under the ear of the infant, behind the jaw, on the side of the face that would face away from the mother during the meal. The longitudinal axis of the sensor was perpendicular to the longitudinal axis of the outer ear. Two video cameras (both Samsung HMX-F80) were positioned to record mouth and jaw movements prior to and during the feed; one focused from the side and the other from above the infant's head. Mothers were instructed to begin the meal once the cameras and the jaw sensor were recording and to make the meal as "natural as possible". Mothers could interrupt the meal to burp the infant or to change positions, as needed, and recording continued during this time. Meals ended when the infant fell asleep, finished the bottle (if applicable), refused to consume more, and/or mothers indicated that the infant finished feeding. Therefore, a meal was defined as the duration between the start and end of the feeding episode of the infant. After burping, infants were reweighed on the PeaPod® scale wearing the same diaper in which they were weighed prior to the meal, and bottles were also reweighed as appropriate. Any milk lost through spillage or regurgitation was captured in pre-weighed burp cloths so that milk consumption could be corrected for spillage. The total size of the recorded dataset for all 10 infants was approximately two hours.

\subsection{Body Composition Assessment}

Body composition was measured by air displacement plethysmography (PeaPod®; Life Measurement Instruments, Concord, CA). In brief, after infant length was measured, infants were weighed on the PeaPod $®$ scale while wearing only a tightly fitted stocking cap to compress their hair. They were then placed inside the test chamber where infant volume was assessed. Infant fat mass and fat free mass were calculated using a two compartment model [23].

\subsection{Training for Coding Discrete Infant Feeding Behaviors: Sucking Count}

A researcher with expertise in coding infant behaviors trained two human raters to code the videotapes of the infant feeding sessions. Each human rater met individually with the researcher for the training. The human raters were informed that they were assisting with testing the reliability of a jaw motion sensor designed to record feeding behaviors. At the training session, video segments of infants being breast-fed and bottle-fed were played while the researcher pointed out discrete examples of infant sucks. A discrete suck was defined as one complete down and up jaw movement when the infant's lips were wrapped around the nipple of the mother or the bottle. The researcher counted out loud the discrete sucks while watching segments of the videotapes with the rater. The rater then performed the same task, counting discrete sucks aloud during several breastand bottle-fed video segments, which the researcher observed. After several trials of 
counting discrete sucks out loud, the researcher and the rater each viewed new video segments of breast- and bottle-fed infants with each independently and quietly counting the number of sucks and recording their total sucks on paper. The total sucks were then examined and a criterion of $90 \%$ agreement with the researcher was established for the rater to be deemed reliable in counting discrete sucks. For example, if the rater noted that a breast-fed infant sucked 120 times in a segment of video and the researcher counted 110 sucks for the same segment $(110 / 120=91.6 \%$ agreement $)$, then the rater was deemed reliable for that segment.

\subsection{Jaw Motion Sensor}

The sensor data collection system consisted of a jaw motion sensor, a data acquisition device, and an Android smart phone. The sensor system was originally designed to be used in adults [17] and was used in this study without any modification. The jaw motion sensor was a piezoelectric film element (DT2-028K; Measurement Specialties Inc. VA, USA). Jaw movements during the sucking process bend the sensor and create an electrical signal proportional to the amount of bending. The signal from the sensor was buffered and amplified by an operational amplifier and then digitized by the microcontroller of the Automatic Ingestion Monitor (AIM) [17] at a sampling frequency of $1 \mathrm{kHz}$. Digitized signals were then transmitted via Bluetooth wireless connection in real time to an Android phone that stored the signal on an SD-card for further processing. Figure 1 shows the jaw motion sensor (Figure 1(a)) and the AIM module (Figure 1(b)); the Android phone is not shown in the picture. Figure 1(c) shows a bottle-fed infant wearing the sensor.

\subsection{Coding of Videotapes}

After the training, video recordings and time-synchronous jaw sensor signals were annotated by the two trained raters independently using a modified version of the software introduced in [21]. To provide synchronization between the sensor signal and the video, data collection was started on the Android phone when it was in view of one of the cameras. To maintain homogeneity of the scoring process, a protocol similar to other studies [24, 25] was developed to mark meal initiation and termination and
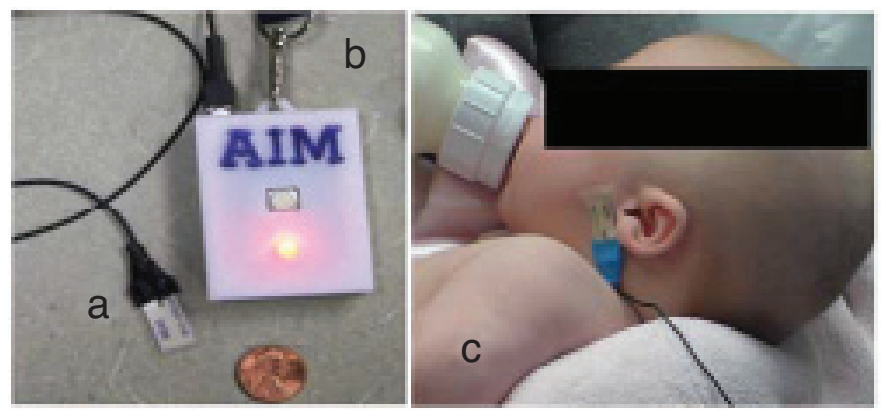

Figure 1. Sensor system for monitoring jaw movements: (a) jaw motion sensor, (b) wireless module, and (c) a bottle-fed infant wearing the sensor. 
sucking count. Human raters marked the start and end of the meal in the video. The period between the start and end of the meal was divided into 10 second epochs (a total of $M$ epochs) in the scoring software and a sucking count was computed for each epoch. The 10 second interval was chosen because in the pilot coding, it was deemed a manageable period to count discrete sucks. These epochs also provided cluster data for conducting intra-class correlation (ICC) analysis between the human raters, and between the human raters and the count provided by the jaw sensor algorithm. Epochs containing partial intake (i.e., video segments where the jaw was not visible in the video) or no intake (including burping, rest period, periods where the nipple was out of the mouth, etc.) were discarded. For the $n$th epoch, the average sucking count from both human raters was used as the annotated per-epoch sucking count, denoted by $A_{C N T}(n)$. On average, the raters counted 664 sucks per meal.

\subsection{Sensor Data and Signal Processing}

Jaw motion (JM) signals retrieved from the sensor (denoted as $J M(t)$ ) were processed in the following manner. First, the signals were demeaned by subtracting the average computed over the duration of the experiment from each data point. This was done to account for signal variation among infants. Next, all signals were de-noised using wavelet transform. De-noising attenuates small variations in the signal due to noise on the power lines (i.e., variation in voltage due to wireless transmission that leaks into the sensor signal). A bi-orthogonal wavelet transform (with Haar mother wavelet) using a lifting scheme was used for de-noising. The lifting scheme allows custom design and fast implementation of the wavelet transform by using the similarities between low and high pass filters [26]. The sensor signal was decomposed using a Discrete Wavelet Transform (DWT) technique. The wavelet coefficients below a threshold $T$ (described below) were discarded and the de-noised sensor signal was recovered using an Inverse Discrete Wavelet Transform. Figure 2 shows a segment of the jaw motion sensor signal before and after de-noising.

\subsection{Sucking Count and Error Computation for Sensor Signal}

After de-noising $J M(t)$, sensor signals were divided into $M$ epochs of 10 second each, denoted as $x(n)$, with $N=10000$ samples per epoch, where $N=L * f, L=10$ second or the epoch size and $f=1000 \mathrm{~Hz}$ (the sampling frequency). These $M$ epochs were timesynchronous with the 10 second epochs used during the signal annotation process. For each epoch, sucking count was computed from the sensor signal by the algorithm shown in Figure 3. The algorithm computed the number of mean crossings $M C(n)$ for each epoch, assuming that the de-noised signal is smooth (Figure 2). Given that a suck was defined as one complete down-up jaw motion, it was also assumed that the number of sensor-determined sucks $P_{C N T}(n)$ is equal to the half of the number of mean crossings. Per-epoch sucking frequency was calculated by dividing number of sucks by the epoch size. Since errors computed for both sucking count and sucking frequency were the same, only results for sucking counts are reported here.

To evaluate the performance of the algorithm, the sensor-determined counts $P_{C N T}(n)$ were compared to human-annotated counts $A_{C N T}(n)$ within each epoch. Two types of 
(a)

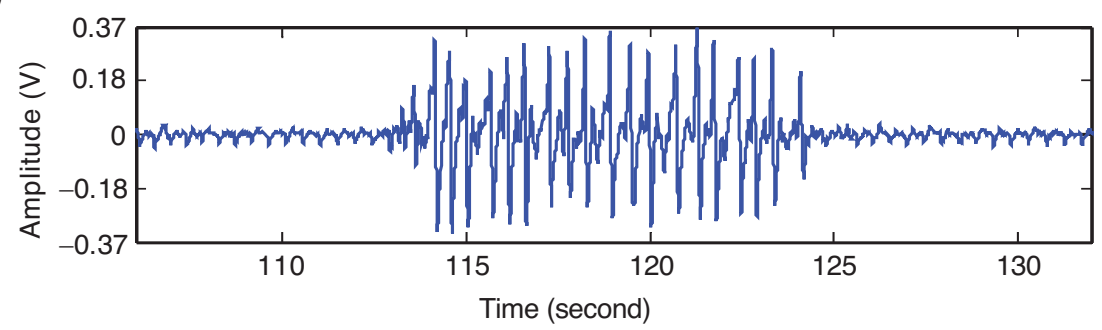

(b)

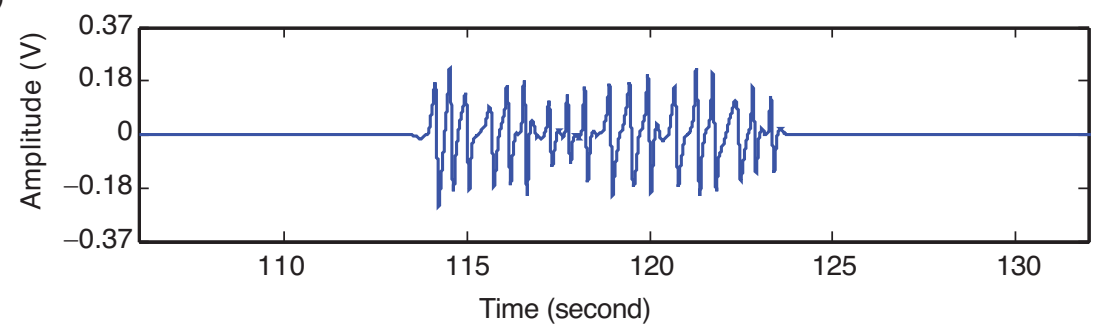

Figure 2. Jaw motion sensor signal before (a) and after (b) de-noising using the bi-orthogonal wavelet transform.

- Set the variable $M C(n)$ (number of mean crossings) to 0 .

- For $i$-th sample in $n$-th epoch, increament $M C(n)$ by 1 , if $[x(i)>\overline{x(n)}$ and $x(i+1)<\overline{x(n)}]$ or $[x(i)>\overline{x(n)}$ and $x(i+1)>\overline{x(n)}]$ where $i=1,2, \ldots \ldots, N$ and $\overline{x(n)}$ is the mean amplitude of the epoch $x(n)$.

- Compute per-epoch sucking count $P_{C N T}(n)$ as $P_{C N T}(n)=M C(n) / 2$

Figure 3. Algorithm for computation of sensor-determined sucking count $\left(P_{C N T}(n)\right)$ for the $n t h$ epoch.

errors were computed for each infant. The first error characterized the per-epoch accuracy in the sucking count, thus illustrating the ability of the sensor to follow temporal changes in the sucking patterns.

For each infant $k$, per-epoch mean error for sucking count $E_{E P O C H}^{C N T}(k)$ was defined as:

$$
E_{E P O C H}^{C N T}(k)=\frac{1}{M} \sum_{n=1}^{M}\left[\frac{\left(A_{C N T}(n)-P_{C N T}(n)\right) * 100}{A_{C N T}(n)}\right]
$$

where $M$ is the total number of epochs, $A_{C N T}(n)$ is annotated suck count for epoch $n$, and $P_{C N T}(n)$ is sensor-determined suck count for epoch $n$. 
The second type of error characterized overall accuracy across the entire feeding episode. The cumulative sucking count error over a meal for infant $k$ was computed as the percent difference between the total counts of annotated sucks versus sensordetermined sucks:

$$
E^{C N T}(k)=\frac{\left[\sum_{n=1}^{M} A_{C N T}(n)-\sum_{n=1}^{M} P_{C N T}(n)\right] * 100}{\sum_{n=1}^{M} A_{C N T}(n)}
$$

\subsection{Parameter Determination and Validation}

The experimental data indicated that the amplitude of the sensor signal was different for each infant (as it depended on the sensor location, infant's strength of sucking, etc.); therefore, the threshold value $T$ used in the de-noising algorithm had to be individually adjusted. As a generalizable approach, the threshold used in de-noising was computed as a function of the jaw sensor signal's amplitude: $T=\alpha * \operatorname{STD}(J M(t))$, where STD is the standard deviation of the signal over the meal duration. A leave-one-out cross validation scheme was used to find the value of the scaling factor $\alpha$ by withholding infant $k$ from the dataset and performing a grid search for a value of $\alpha \quad[1,10]$ on the dataset from the remaining 9 infants (training set). The value of $\alpha$ which resulted in the minimal absolute average $E_{E P O C H}^{C N T}$ on the training set, was used to validate performance of the method on the withheld (validation) data of infant $k$ by computing corresponding $E_{E P O C H}^{C N T}(k)$ and $E^{C N T}(k)$. This process was repeated such that each infant was used for validation only once.

During algorithm development, there were two possibilities for finding the threshold and for parameter selection. One possibility was to optimize the per-epoch sucking count error $E_{E P O C H}^{C N T}(k)$ and the other possibility was to optimize the cumulative sucking count error $E^{C N T}(k)$. Per-epoch sucking count provides a better estimate of the feeding behavior during a meal compared to the cumulative sucking count and therefore it was chosen for parameter optimization.

Measures of sample-wide performance were then computed as average values of $E_{E P O C H}^{C N T}(k)$ and $E^{C N T}(k)$ over validation results from all 10 infants:

$$
\begin{gathered}
\overline{E_{E P O C H}^{C N T}}=\frac{1}{10} \sum_{k=1}^{10} E_{E P O C H}^{C N T}(k) \\
\overline{E^{C N T}}=\frac{1}{10} \sum_{k=1}^{10} E^{C N T}(k)
\end{gathered}
$$

These two sample-based errors give performance estimation of the proposed technique for predicting both per-epoch as well as cumulative values of sucking count across the whole sample and are used as the main metrics of performance. 


\subsection{Intra-Class Correlation (ICC) and Statistical Analysis}

Intra-class correlation (ICC) analyses were conducted to examine the reliability between the two human raters, and the reliability between the human raters (averaged together) and the sensor-determined sucking counts. In both cases, a 2-way mixed model with fixed observers and random subjects was used for analysis.

Infant weight-for-age, length-for-age, and body mass index-for-age z-scores were calculated using the World Health Organization (WHO) Anthro software (version 3.2.2, January 2011), which is based on international growth charts of healthy infants growing under optimal conditions [27]. Exploratory analyses were conducted to examine whether any other individual factors influenced the accuracy of the sensor-determined sucking counts. Two-tailed, two-sample t-tests were used to examine the effect of feeding mode (breast versus bottle) and gender on the error in the sensor-determined sucking counts. A Passing-Bablok regression [28] was used to determine the effects of body size (i.e., BMI-for-age z-score, weight-for-age z-scores, length-for-age z-scores), age, and body composition (i.e., \% body fat, total fat mass and total fat free mass) on the prediction error. Passing-Bablok regression is insensitive to outliers and assumes that measurement errors in both variables have the same distribution. All analyses were conducted using MATLAB® (Mathworks Inc.).

\section{RESULTS}

The sample consisted of 5 male and 5 female infants with the racial distribution of 6,2 , and 2 of Caucasian, African American and other (mixed race), respectively. Age of the male and female infants did not differ $(16.44 \pm 5.00$ weeks for males and $16.35 \pm 2.93$ weeks for females), but male infants showed a trend towards consuming more during the test meal than female infants $(130.00 \pm 63.94 \mathrm{ml}$ versus $58.50 \pm 30.65 \mathrm{ml}$, respectively; $p=0.10)$. The sample consisted of 6 breast-fed and 4 bottle-fed infants. Breast-fed infants showed a trend towards consuming less than the bottle-fed infants $(74.41 \pm$ $28.39 \mathrm{ml}$ versus $140.12 \pm 69.05 \mathrm{ml}$, respectively; $p=0.07$ ). The average gestational age at birth was $39.9 \pm 1.5$ weeks and average birth weight was $3.6 \pm 0.3 \mathrm{~kg}$.

There were a total of 692 epochs in the data set. The ICC analysis of the sucking count between the two raters showed a correlation coefficient of 0.98 [95\% CI: 0.98 , 0.99]. The ICC analysis between the raters (averaged together) and the sensordetermined count showed a correlation coefficient of 0.86 [95\% CI: $0.83,0.88$ ].

With respect to the accuracy of the sensor-determined per-epoch sucking count, the sensor-based method resulted in a mean error of $\overline{E_{E P O C H}^{C N T}}=-9.72 \pm 20.03 \%$, and the cumulative sucking count error $\overline{E^{C N T}}=-2.38 \pm 22.33 \%$ for the entire meal. Per-infant errors are summarized in Table 1. This table also provides the mean absolute errors. Figure 4 shows an example of the annotated and the sensor-determined sucking count for an infant over the period of an entire experiment.

A comparison of the per-epoch mean error in sucking count was performed between bottle-fed and breast-fed infants. The results of the t-test showed that the per-epoch mean errors were greater among the bottle-fed than the breast-fed infants $(-26.62$ $\pm 14.16 \%$ for bottle-fed infants and $1.54 \pm 14.88 \%$ for breast-fed infants; with $p=0.02$ ). 


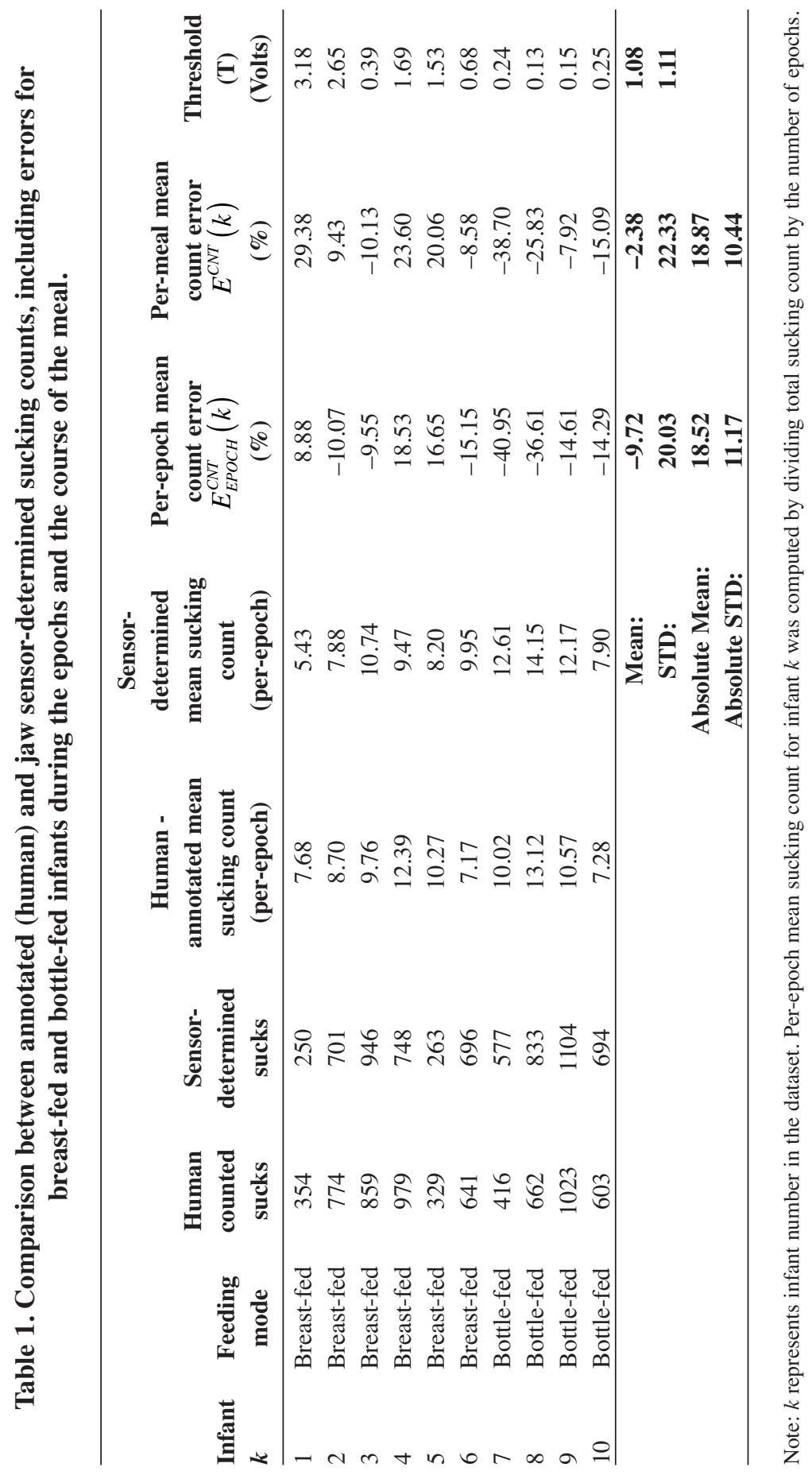




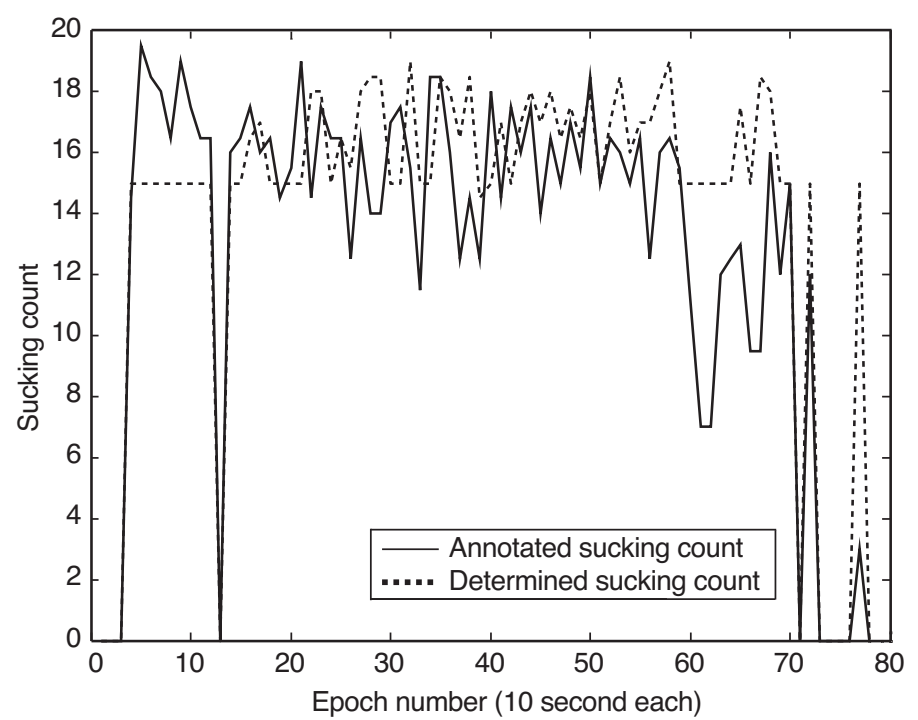

Figure 4. A comparison of human-annotated sucking count vs. sensor-determined sucking count for the duration of the entire experiment (infant \#9). Epochs where both values are zeros are the epochs that involved no intake.

A comparable analysis between male and female infants showed no difference in perepoch mean error $(-17.76 \pm 23.59 \%$ for males, $-1.68 \pm 13.59 \%$ for females; with $p=0.22$ ).

Results of Passing-Block regression analyses to examine whether the per-epoch sucking count error was independent of body size and age are shown in Figures 5(a) through 5(d). Figures 6(a) through 6(c) show the results of the Passing-Block regression analyses to examine whether the per-epoch sucking count error was independent of body composition. In all analyses, results suggested that the error may be independent of these individual factors.

\section{DISCUSSION}

Previous studies showed that feeding behavior and weight gain during the first 6 months of infancy are associated with risk for obesity later in life. Therefore, it is important to accurately monitor feeding behavior in infants. The assessment of infant sucking count during meals is a useful first step to develop a method to measure feeding behavior in infants because this parameter can be used to determine sucking frequency (rate), which has been shown to associate with obesity, and other feeding-related parameters such as meal duration and frequency. In this study, we introduced a new approach to objective monitoring of infant sucking using a jaw motion sensor. The jaw sensor system was tested on a sample consisting of both breast-fed and bottle-fed infants to examine its feasibility for both feeding modes. The study compared the number of sucking counted 
(a)

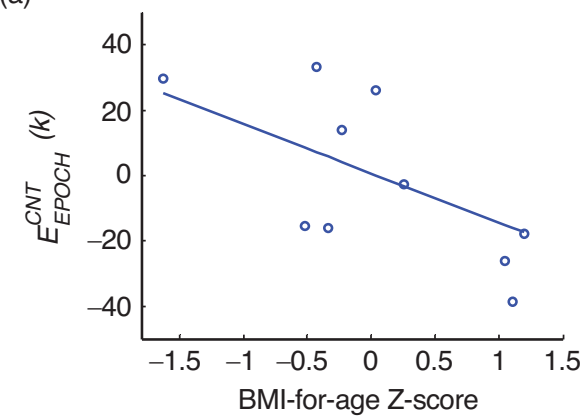

(c)

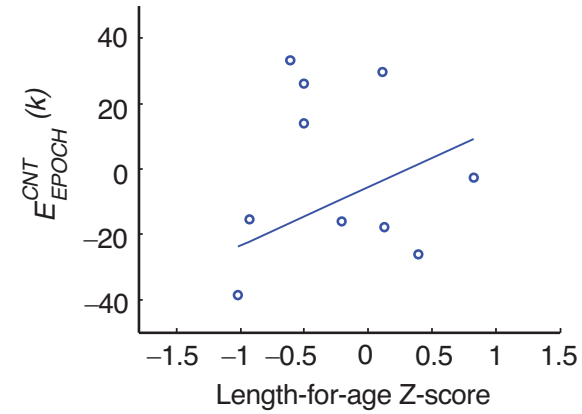

(b)

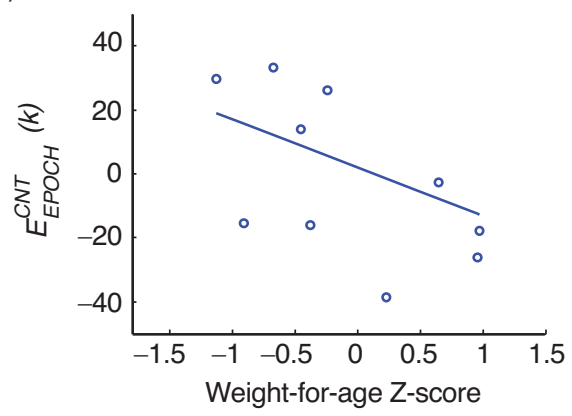

(d)

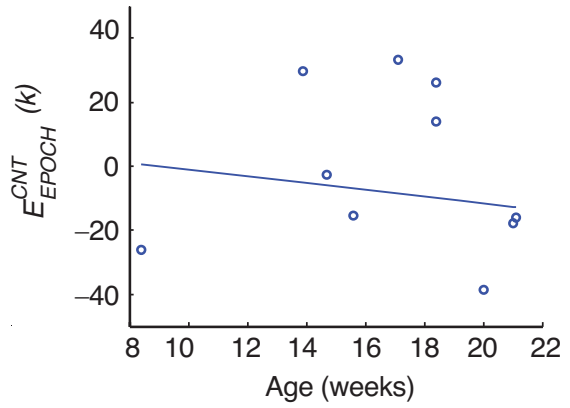

Figure 5. (a) Passing-Bablok regression analysis for $E_{E P O C H}^{C N T}(k)$ and BMI-for-age Zscore. Regression line equation $\mathrm{y}=-0.01-0.15 \mathrm{x} ; 95 \% \mathrm{CI}$ is -0.06 to 0.06 for intercept and is -0.34 to 0.23 for slope. (b) Passing-Bablok regression analysis for $E_{E P O C H}^{C N T}(k)$ and Weight-for-age Z-score. Regression line equation $\mathrm{y}=0.02-0.15 \mathrm{x} ; 95 \% \mathrm{CI}$ is -0.02 to 0.03 for intercept and is -0.32 to 0.28 for slope. (c) Passing-Bablok regression analysis for $E_{E P O C H}^{C N T}(k)$ and Length-for-age Z-score. Regression line equation $\mathrm{y}=-0.06-0.18 \mathrm{x} ; 95 \% \mathrm{CI}$ is -0.05 to 0.46 for intercept and is -0.1733 to 1.25 for slope. (d) PassingBablok regression analysis for $E_{E P O C H}^{C N T}(k)$ and age (in weeks). Regression line equation $\mathrm{y}=0.09-0.01 \mathrm{x} ; 95 \% \mathrm{CI}$ is -0.81 to 2.23 for intercept and is -0.12 to 0.05 for slope.

by human raters to that determined by the jaw sensor. The method used to annotate the sucking count by observation (i.e., human raters) was found to be highly reliable (correlation coefficient $=0.98$ ) between two independent raters. Although the reliability between the average of the human raters' sucking count and that determined by the jaw sensor and the signal algorithm was a little lower, it still indicated a strong agreement (correlation coefficient $=0.86$ ), suggesting that the jaw sensor may be a reliable method to monitor sucking count. The error in the sucking count determined by the jaw sensor was approximately $10 \%$ and $3 \%$, for per-epoch and per meal, respectively. Errors were 
(a)

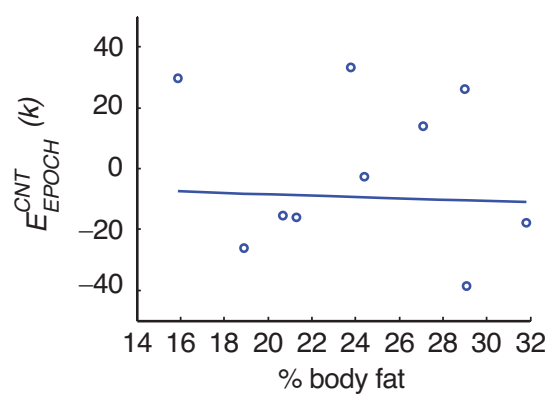

(b)

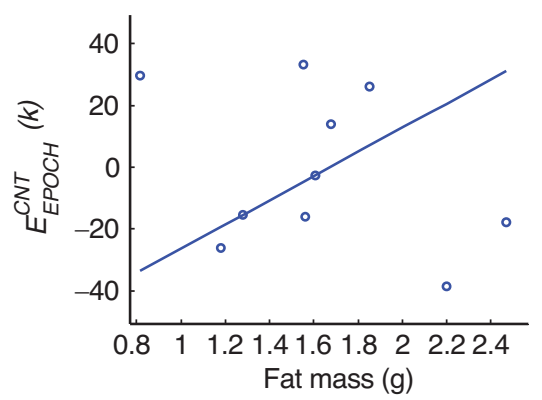

(c)

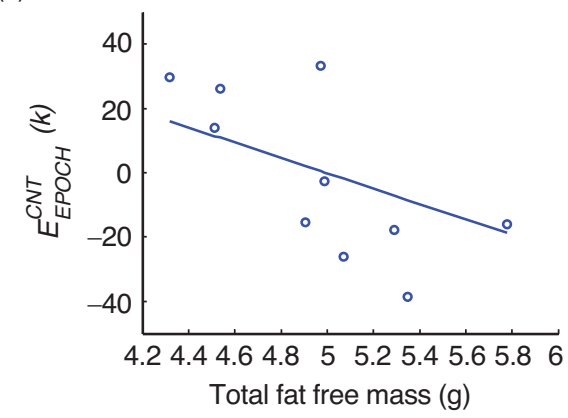

Figure 6. (a) Passing-Bablok regression analysis for $E_{E P O C H}^{C N T}(k)$ and $\%$ body fat. Regression line equation $\mathrm{y}=-0.03-0.01 \mathrm{x}$; $95 \% \mathrm{CI}$ is -1.25 to 1.17 for intercept and is -0.05 to 0.05 for slope. (b) Passing-Bablok regression analysis for $E_{E P O C H}^{C N T}(k)$ and Total fat mass (in $\mathrm{g}$ ). Regression line equation $\mathrm{y}=-0.65+0.39 \mathrm{x} ; 95 \% \mathrm{CI}$ is -2.57 to 0.38 for intercept and is -0.23 to 1.60 for slope. (c) Passing-Bablok regression analysis for $E_{E P O C H}^{C N T}(k)$ and Total fat free mass (in $\mathrm{g}$ ). Regression line equation $\mathrm{y}=1.19-0.24 \mathrm{x} ; 95 \%$ CI is -2.67 to 2.99 for intercept and is -0.61 to 0.52 for intercept.

in the negative direction, suggesting that the jaw sensor method over-estimated the sucking count. Although promising, the relatively high error, particularly for some infants, and the high standard deviation indicate variation in the performance of the sensor-algorithm system among infants. Figure 4 shows that the count determined by the jaw sensor followed the trend of the human rater's count, but modestly overestimated in most cases. It is possible that the disagreement may be attributable to the presence of non-nutritive sucking periods, or to body movements (motion artifacts) that can increase the sensor-determined sucking count. It is also possible that small sucks were detected by the sensor but not by the human raters because the chin movement 
generated by the suck was almost imperceptible. These potential sources of error will be the subject of further investigation. The addition of other sensor modalities such as monitoring of swallowing may help in eradicating false positives.

One advantage of the proposed method is its potential to study the feeding behaviors for both breast-fed and bottle-fed infants. Although other sensors that are placed on the mother or the bottle may be useful, to our knowledge, no previous study has attempted to use the same sensor to examine both feeding modes. Preliminary results from this cohort suggest that the sensor generated a higher per-epoch mean error (as well as standard deviation) for bottle-fed infants compared to breast-fed infants (i.e., the technique performed better for breast-fed infants compared to bottle-fed infants). Although not examined here, it is possible that differences in infant's body movements during the meal may have contributed to greater error among bottle-fed infants, or that bottle-fed infants engage in more non-nutritive sucking during the meal in comparison to breast-fed infants. An alternate explanation may be that breast-fed infants have to open the mouth wider and have more pronounced jaw movements since suckling from a breast requires more effort than sucking from a bottle nipple where milk flow may be induced by gravity (milk level always maintained above mouth lest the infant should suck air), and thus breast-fed infants' jaw movements were more perceptible by the sensor. The source of this feeding mode variation in the accuracy of the method needs further investigation in a larger sample. Another possibility may be to analyze the strength/amplitude of the sensor signal for bottle-fed and breast-fed infants and implement different processing for each group.

Other individual factors that were included in this study to examine their potential effect on the performance of the sensor were gender, age, and indices of body size and body composition. It was important to explore the influence of these variables because infant body size, composition, and therefore, function, change rapidly during the first six months of life. Our results showed that there was no gender difference in the error between the sensor and human rater methods, and there was no association between the sensor performance (i.e., error) and infant age, or indices of infant size and body composition. It is important to note, however, that the small sample size may have limited the power of this study to detect any association. These results, therefore, are provided as an initial consideration of potential influences on sensor performance and would need to be replicated in a larger cohort before a conclusion could be drawn.

The small sample size is the main limitation of this study and, therefore, results should be interpreted with caution until further research results are available. Despite this limitation, this study is an important first step in using a jaw sensor-based approach for the investigation of nutritive sucking in both breast-fed and bottle-fed infants, and results are supportive of the feasibility of this method to objectively monitor the feeding behavior of infants. Future work will involve larger cohorts to more comprehensively examine the performance of the sensor. Another possible limitation is that the sensor used in these experiments was originally designed to be used by adults and so the size may not be optimal for infants. In this cohort, however, there was no indication that the sensor size caused any discomfort to the infants or mothers, and the sensor did not impede bottle or breast feeding. Nonetheless, a smaller sensor may help to reduce 
over-estimation of the sucking count, and such an adaptation is technologically possible by incorporating the electronics of the sensor system into the infant's clothing [29]. It is also important to note that there was no fixed bottle position for bottle-fed infants. Visual analysis of the videos showed that the bottles were angled somewhere between 0 (perfectly horizontal) and 90 degrees (perfectly vertical). Although no analysis was done to examine whether gravity induced milk to drop into the infants' mouth without effort, visual inspection suggested active sucking from the infants to receive milk. Effects of bottle nipple size were not investigated in this study. It is also important to acknowledge that the experiments of this study were performed under laboratory conditions, and free-living tests will be required to evaluate performance of the proposed technique over extended periods of time under realistic conditions of daily living. An alternate approach to monitor feeding among infants is by recording pre- and post-meal body weights. Although well established, this method is also subject to error in the home environment and is useful only to measure overall intake, rather than within-meal feeding behavior. The jaw motion sensor may provide a complementary method to assess infant feeding behavior, or could potentially be developed further into a fully automatic version that is able to detect meal periods and energy intake, along with other useful behavioral parameters such as meal duration and frequency, etc.

Future studies might also examine whether meal and sucking burst boundaries (the start and the end of the episodes of continuous sucking) can be automatically recognized. In the current study, meals were marked by human raters. The relative strength of the sucking signal and the frequency of swallowing may provide indicators suitable for differentiation of food-related sucking with sucking on thumbs, pacifiers, and vocalizing, etc. The strength of the sucking signal may also provide another index of interest that might relate to obesity.

Overall, the results of this study suggest that monitoring of jaw motion has the potential to provide an objective measure of infant feeding behavior.

\section{CONCLUSION}

This paper evaluated the technical feasibility of using a jaw motion sensor for accurate and objective monitoring of feeding behavior among bottle-fed and breast-fed infants. The signals captured by the sensor were processed to estimate sucking counts. The computed counts were compared with the results of human annotation of the same feeding episodes. The mean errors and ICC statistics showed a close, and acceptable, agreement between the human raters and the proposed sensor methodology for collecting data on infant sucking behavior during a meal. Statistical analysis of the results suggests that the performance of the proposed method is independent of factors such as gender, BMI, length, weight and age of the infants, but it did differ by the feeding mode. Further study with a larger sample is needed to more rigorously examine the statistical significance of these results. This would also ensure the applicability of the proposed method to a wider infant population. The preliminary findings suggest the potential of the sensor as a novel, effective and objective tool for monitoring infant feeding behavior. 


\section{ACKNOWLEDGEMENTS}

This work was supported by a pilot grant from Nutritional Obesity Research Center at the University of Alabama, Birmingham (UAB). The authors would like to acknowledge the efforts of infants and mothers who participated in the study as well as students who took part in conducting the experiment and annotating the data.

\section{CONFLICT OF INTEREST}

The authors have no potential conflicts of interest.

\section{NOMENCLATURE}

$A_{C N T}$
$E_{E P O C H}^{C N T}$
$E^{C N T}$
$\overline{E^{C N T}}$
$\overline{E_{E P O C H}^{C N T}}$
$f$
$J M$
$L$
$M$
$M C$
$N$
$P_{C N T}$
$T$
$x$

Annotated sucking count (number of sucking)

Per-epoch mean error for sucking count for each subject

Cumulative sucking count error over all epochs for each subject

Population-wide mean error for sucking count including all subjects.

Population-wide mean per-epoch error for sucking count including all subjects

Greek

\section{REFERENCES}

[1] Hester SN, Hustead DS, Mackey AD, Singhal A, Marriage BJ. Is the macronutrient intake of formulafed infants greater than breast-fed infants in early infancy? Journal of Nutrition and Metabolism. 2012, 891201.

[2] Mihrshahi S, Battistutta D, Magarey A, Daniels LA. Determinants of rapid weight gain during infancy: baseline results from the NOURISH randomised controlled trial. BMC Pediatrics. 2011, 11(1):99.

[3] Gillman MW, Rifas-Shiman SL, Camargo, Jr CA, Berkey CS, Frazier AL, Rockett HR, Colditz GA. Risk of overweight among adolescents who were breastfed as infants. Journal of the American Medical Association. 2001, 285(19):2461-2467.

[4] Wright P, Fawcett J, Crow R. The development of differences in the feeding behaviour of bottle and breast fed human infants from birth to two months. Behavioural Processes. 1980, 5(1):1-20. 
[5] Taki M, Mizuno K, Murase M, Nishida Y, Itabashi K, Mukai Y. Maturational changes in the feeding behaviour of infants - a comparison between breast-feeding and bottle-feeding. Acta Pcediatrica. 2010, 99(1):61-67.

[6] Ojha S, Saroha V, Symonds ME, Budge H. Excess nutrient supply in early life and its later metabolic consequences. Clinical and Experimental Pharmacology \& Physiology. 2013, 40(11):817-823.

[7] Yang Z, Huffman SL. Nutrition in pregnancy and early childhood and associations with obesity in developing countries. Maternal \& Child Nutrition. 2013, 9 Suppl 1105-119.

[8] Dubois L, Girard M. Early determinants of overweight at 4.5 years in a population-based longitudinal study. International Journal of Obesity. 2006, 30(4):610-617.

[9] Stettler N, Zemel BS, Kumanyika S, Stallings VA. Infant Weight Gain and Childhood Overweight Status in a Multicenter, Cohort Study. Pediatrics. 2002, 109(2):194-199.

[10] Taveras EM, Rifas-Shiman SL, Sherry B, Oken E, Haines J, Kleinman K, Rich-Edwards JW, Gillman MW. Crossing growth percentiles in infancy and risk of obesity in childhood. Archives of Pediatrics \& Adolescent Medicine. 2011, 165(11):993-998.

[11] Ekelund U, Ong K, Linné Y, Neovius M, Brage S, Dunger DB, Wareham NJ, Rössner S. Upward weight percentile crossing in infancy and early childhood independently predicts fat mass in young adults: the Stockholm Weight Development Study (SWEDES). The American Journal of Clinical Nutrition. 2006, 83(2):324-330.

[12] Eriksson M, Tynelius P, Rasmussen F. Associations of birthweight and infant growth with body composition at age 15 - the COMPASS study. Paediatric and Perinatal Epidemiology. 2008, 22(4):379-388.

[13] Oyama M, Saito T, Nakamura K. Rapid weight gain in early infancy is associated with adult body fat percentage in young women. Environmental Health and Preventive Medicine. 2010, 15(6):381-385.

[14] Weber F, Woolridge MW, Baum JD. An ultrasonographic study of the orgenisation of sucking and swallowing in newborn infants. Pediatric Research. 1984, 18(8):806-806.

[15] Stunkard AJ, Berkowitz RI, Stallings VA, Schoeller DA. Energy intake, not energy output, is a determinant of body size in infants. The American Journal of Clinical Nutrition. 1999, 69(3):524-530.

[16] Da Costa SP, van der Schans CP, Boelema SR, van der Meij E, Boerman MA, Bos AF. Sucking patterns in fullterm infants between birth and 10 weeks of age. Infant Behavior and Development. 2010, 33(1):61-67.

[17] Sazonov E, Fontana JM. A Sensor System for Automatic Detection of Food Intake Through NonInvasive Monitoring of Chewing. IEEE Sensors Journal. 2012, 12(5):1340-1348.

[18] Amft O. A wearable earpad sensor for chewing monitoring. In: 2010 IEEE Sensors. 2010. p. 222-227.

[19] Fontana JM, Sazonov ES. A robust classification scheme for detection of food intake through noninvasive monitoring of chewing. Conf Proc IEEE Eng Med Biol Soc. 2012. p. 4891-4894.

[20] Fontana JM, Farooq M, Sazonov E. Automatic Ingestion Monitor: A Novel Wearable Device for Monitoring of Ingestive Behavior. IEEE Transactions on Biomedical Engineering, 2014, 61(6):1772-1779.

[21] Sazonov E, Schuckers S, Lopez-Meyer P, Makeyev O, Sazonova N, Melanson EL, Neuman M. Noninvasive monitoring of chewing and swallowing for objective quantification of ingestive behavior. Physiological Measurement. 2008, 29(5):525-541.

[22] Use of World Health Organization and CDC Growth Charts for Children Aged 0-59 Months in the United States. http://www.cdc.gov/mmwr/preview/mmwrhtml/rr5909a1.htm Accessed: Dec 19, 2013.

[23] Fomon SJ, Haschke F, Ziegler EE, Nelson SE. Body composition of reference children from birth to age 10 years. The American Journal of Clinical Nutrition. 1982, 35(5):1169-1175.

[24] Hernandez-Reif M, Field T, Diego M. Differential sucking by neonates of depressed versus nondepressed mothers. Infant Behavior and Development. 2004, 27(4):465-476.

[25] Hernandez-Reif M, Field T, Del Pino N, Diego M. Less exploring by mouth occurs in newborns of depressed mothers. Infant Mental Health Journal. 2000, 21(3):204-210. 
[26] Sweldens W. The Lifting Scheme: A Custom-Design Construction of Biorthogonal Wavelets. Applied and Computational Harmonic Analysis. 1996, 3(2):186-200.

[27] WHO I The WHO Multicentre Growth Reference Study (MGRS): Rationale, planning, and implementation. http://www.who.int/childgrowth/mgrs/fnu/en/. Accessed Dec 8, 2013.

[28] Passing H, Bablok. A new biometrical procedure for testing the equality of measurements from two different analytical methods. Application of linear regression procedures for method comparison studies in clinical chemistry, Part I. Journal of Clinical Chemistry and Clinical Biochemistry. 1983, 21(11):709-720.

[29] Chen W, Bambang Oetomo S, Feijs L, Bouwstra S, Ayoola I, Dols S. Design of an Integrated Sensor Platform for Vital Sign Monitoring of Newborn Infants at Neonatal Intensive Care Units. Journal of Healthcare Engineering, 2010, 1(4):535-553. 



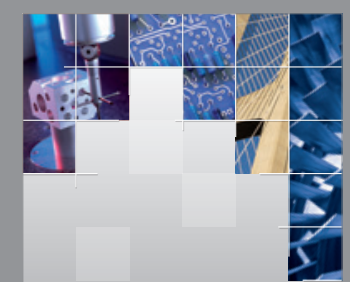

\section{Enfincering}
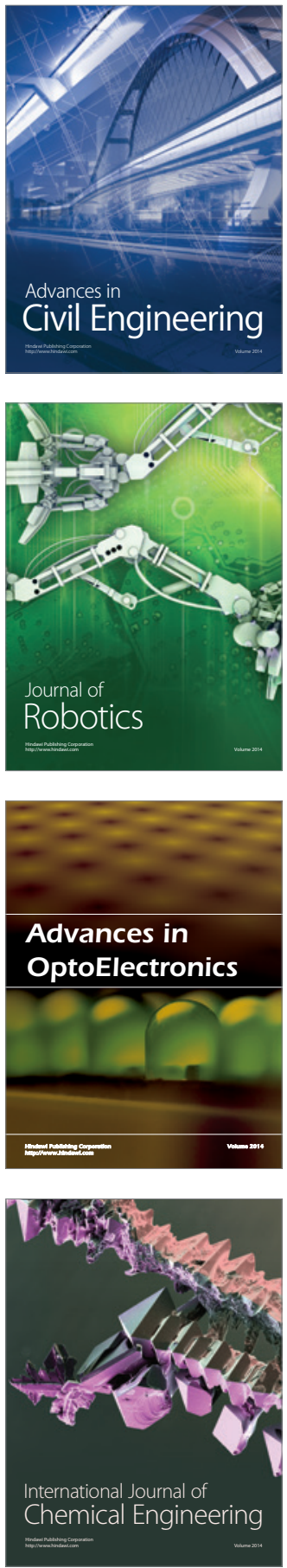

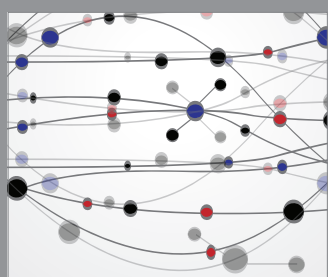

The Scientific World Journal

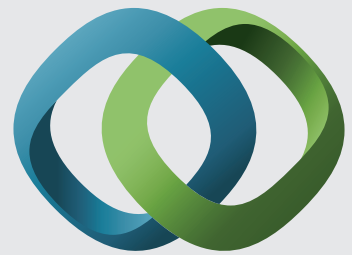

\section{Hindawi}

Submit your manuscripts at

http://www.hindawi.com
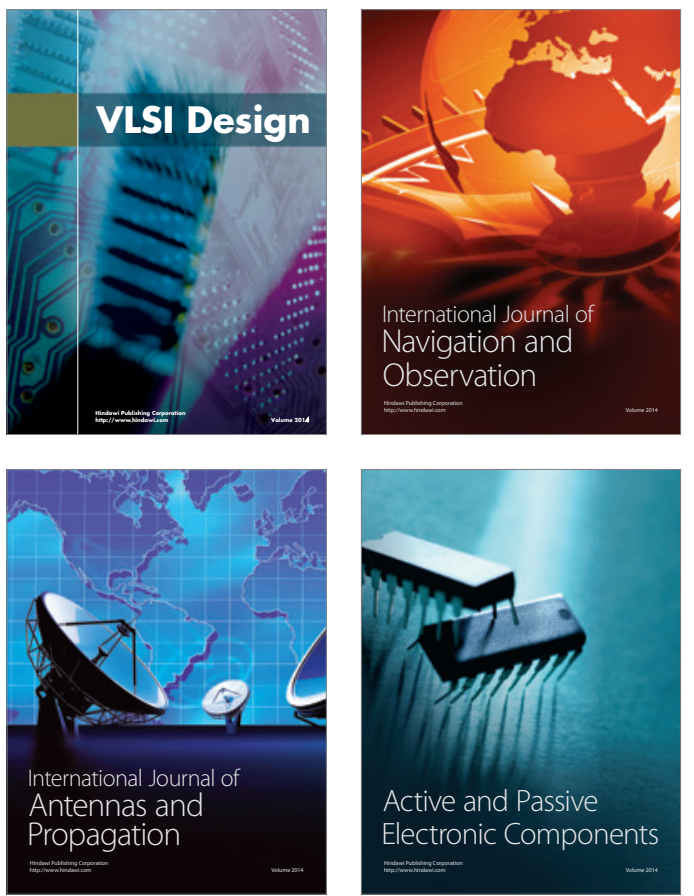
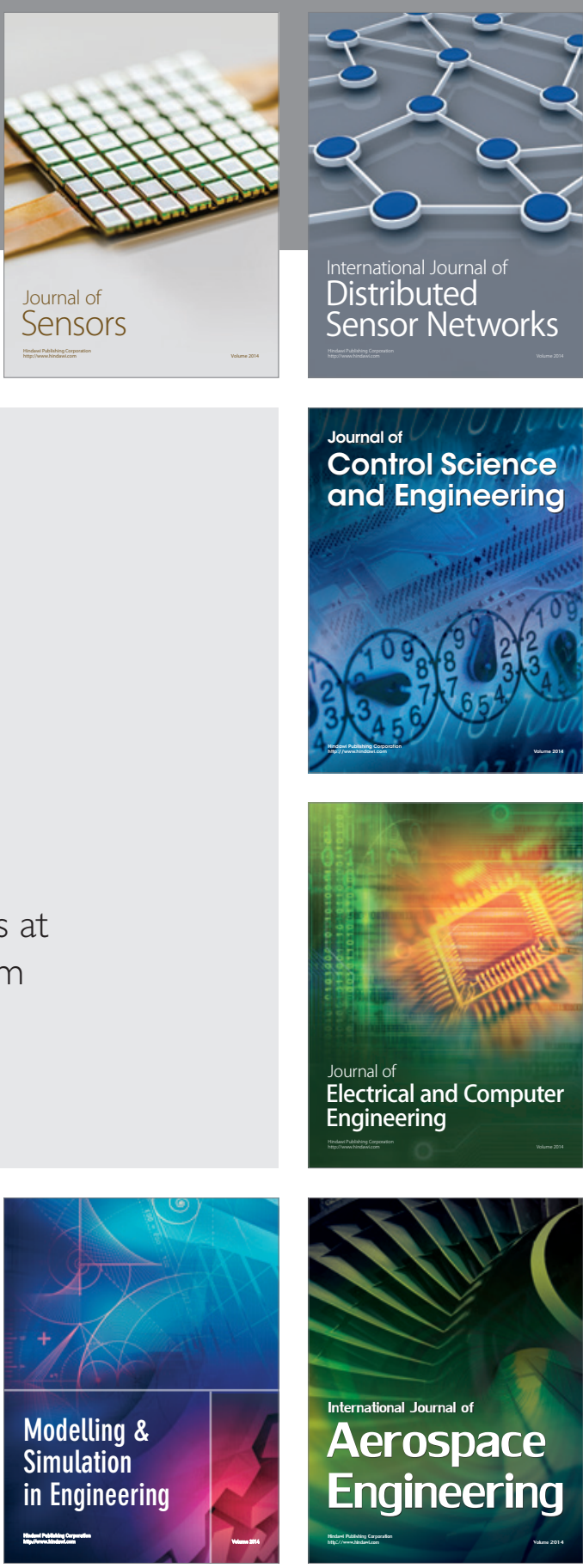

International Journal of

Distributed

Sensor Networks

Journal of

Control Science

and Engineering
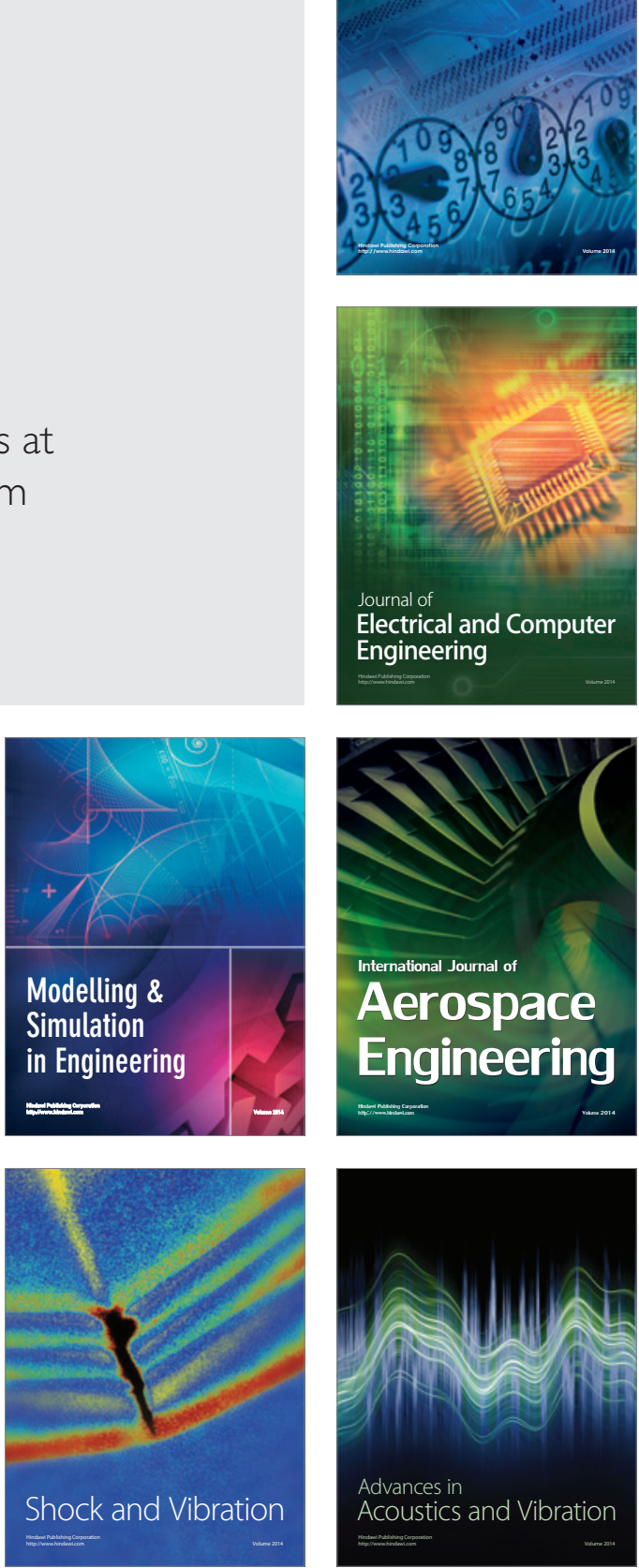